\title{
Analog of the Darboux problem for a loaded integro-differential equation involving the Caputo fractional derivative
}

\author{
U. Baltaeva ${ }^{1,2, *}$, Y. Alikulov ${ }^{3}$, I. I. Baltaeva ${ }^{2}$, A. Ashirova ${ }^{4}$ \\ ${ }^{1}$ Khorezm Mamun Academy, Markaz-1, 220900, Khiva, Uzbekistan \\ ${ }^{2}$ Urgench State University, Kh.Alimdjan str. 14, 220100, Urgench, Uzbekistan \\ ${ }^{3}$ Tashkent University of Information Technologies named after Muhammad Al-Khwarizmi, Amir Temur str. 108, \\ 100200 Tashkent, Uzbekistan \\ ${ }^{4}$ Urganch branch of Tashkent University of Information Technology named after Muhammad al-Khwarizmi, \\ Al Khorezmi str. 110, 220100 Urgench, Uzbekistan \\ umida_baltayeva@mail.ru,yolqin-aliqulov@mail.ru,iroda-b@mail.ru,anorgul@mail.ru
}

PACS 02.30.Jr

DOI 10.17586/2220-8054-2021-12-4-418-424

In this paper, we prove the unique solvability of an analogue problem Darboux for a loaded integro-differential equation with Caputo operator by method of integral equations. The problem is equivalently reduced to a system of integral equations, which is unconditionally and uniquely solvable.

Keywords: integro-differential equations, Caputo fractional derivative, loaded equation, nonlocal problem, Bessel function.

Received: 9 June 2021

Revised: 17 July 2021

\section{Introduction and formulation of the problem}

In recent years, the range of problems under consideration for partial differential equations, as well as for equations of hyperbolic, parabolic and mixed types, has significantly expanded. Along with the study of the main boundary value problems for such equations, since the 1970's, much attention of researchers is paid to the formulation and study of nonlocal boundary value problems. This is explained by the fact that many practically important problems associated with the dynamics of soil moisture, with the process of particle diffusion in turbulent plasma with cooling an inhomogeneous curved rod, modeling the process of laser radiation lead to nonlocal problems [1,2].

In prior research to date, mainly nonlocal boundary value problems for second-order equations have been studied.

Equations of the third and higher order, which, according to similar equations of the second order, have very important applications, were considered in the works of Berdyshev A.S. [3], Sabitov K. B. [4], Zikirov O.S. and Kholikov D. K. [5], Kh. Belakroum, A. Ashyralyev, A. Guezane-Lakoud [6], Khashimov A. R, Smetanová D. [7], as well as in $[8,9]$.

On the other side, in connection with intensive research on problems of optimal control of the agro economical system, long-term forecasting and regulating the level of ground waters and soil moisture, problems of mathematical biology: population dynamics and problems of mathematical economics, it has become necessary to investigate a new class of equations called as "loaded equations". Such equations were investigated for the first time by A. Knezer (1914), L. Lichtenstein (1931). However, they did not use the term "loaded equation". This terminology was introduced by A. M. Nakhushev (1976), where the most general definition of the loaded equation is given and various loaded equations are classified in detail [1].

We can cite by many examples as which emphasized the development of the theory of the loaded equation in the last several decades. As we know [1], mathematical models of nonlocal physics-biological fractal processes represented by loaded integro-differential equations or loaded differential equations especially with integro-differential operators $[1,9]$.

How we know, there has been a significant development in nonlocal problems for fractional differential equations or inclusions (we can see [10] and their literature). Very recently, in the works [11], [12] authors investigated the fractional order integro-differential equations with nonlocal conditions. However, to the best of our knowledge, the nonlocal problems for fractional integro-differential equations in the Caputo derivative sense have not been discussed extensively [10].

Based on the above, the main aim of this paper is to formulate and investigate the unique solvability of a boundaryvalue problem type of Darboux (Cauchy-Goursat) for a third-order loaded partial differential equation of hyperbolic type, with the Caputo operators. For investigating this problem, we reduce the boundary-value problem for the loaded 
equation to a new non-local problem for the fractional order integro-differential equation [8,9]. Taking into account the unique solvability of non-local problems, we prove the solvability of the local boundary-value problem for the loaded equation.

We consider the following linear loaded [13] integro-differential equation:

$$
\frac{\partial}{\partial x}\left(u_{x x}-u_{y y}+a u_{x}+b u_{y}+c u\right)=c D_{0 x}^{\alpha} u(x, 0),
$$

where $c D_{0 x}^{\alpha} f(x)$ is a fractional differential operator of Caputo type [14], $0<\alpha<1, a, b, c$ are given real parameters.

Let $\Omega$ be a characteristic triangle bounded by the segment $A B$ the axis $O X$ and two characteristics

$$
A C: x+y=0, \quad B C: x-y=1
$$

of equation (1) for $y<0, \quad I=\{(x, y): 0<x<1, y=0\}$.

Problem $\mathbf{D}_{1}$. (Darboux) Find a regular solution $u(x, y)$ of the equation (1) in $\Omega$, which is continuous in $\bar{\Omega}$, has continuous derivatives $u_{x}\left(u_{y}\right)$, up to $A B \cup A C$, and satisfies the boundary-value conditions:

$$
\begin{gathered}
\left.u_{y}(x, 0)\right|_{y=0}=\varphi_{1}(x), \quad 0 \leq x<1, \\
u(x,-x)=\psi_{1}(x),\left.\quad \frac{\partial u(x, y)}{\partial n}\right|_{y=-x}=\psi_{2}(x), \quad 0 \leq x \leq \frac{1}{2},
\end{gathered}
$$

where $\varphi_{1}(x), \psi_{1}(x)$ and $\psi_{2}(x)$ are given functions.

\section{Representation of solution of the equation}

One of the most important aspect of the investigation of the boundary-value problem, is shown by the following theorem.

Theorem 1. Any regular solution of equation (1) is represented in the form:

$$
u(x, y)=z(x, y)+w(x),
$$

where $z(x, y)$ is a solution to the equation:

$$
\frac{\partial}{\partial x}\left(z_{x x}-z_{y y}+a z_{x}+b z_{y}+c z\right)=0
$$

$w(x)$ is a solution of the following integro-differential equation

$$
w^{\prime \prime \prime}(x)+a w^{\prime \prime}(x)+c w^{\prime}(x)-c D_{0 x}^{\alpha} w(x)=c D_{0 x}^{\alpha} z(x, 0) .
$$

Proof. Let $z(x, y)$ be a regular solution of the equation (5), $w(x)$ is a solution of the equation (6). Then, we must prove that the function represented by formula (4) will be solution of the equation (1). Thus, in (1), $u(x, y)$ instead of $z(x, y)+w(x)$ taking into account (5) and (6), it is easy to verify that the relation:

$$
\begin{gathered}
\frac{\partial}{\partial x}\left(u_{x x}+a u_{x x}+b u_{y}+c u\right)-c D_{0 x}^{\alpha} u(x, 0)=\frac{\partial}{\partial x}\left(z_{x x}+a z_{x}+b z_{y}+c z\right)+ \\
+w^{\prime \prime \prime}(x)+a w^{\prime \prime}(x)+c w^{\prime}(x)-c D_{0 x}^{\alpha} w(x)-c D_{0 x}^{\alpha} z(x, 0)=0
\end{gathered}
$$

that is, represented by formula (4), is a solution of the equation (1) for $y>0$. Then, vice versa, let $u(x, y)$ be a regular solution of the equation (1), and $w(x)$ be a certain solution of the following integro-differential equation:

$$
w^{\prime \prime \prime}(x)+a w^{\prime \prime}(x)+c w^{\prime}(x)=c D_{0 x}^{\alpha} u(x, 0) .
$$

Let us prove the validity of the relation (4).

Consequently, the function:

$$
u(x, y)=z(x, y)+\left(-\frac{2}{a}\right) \int_{0}^{x}\left(\left(x-t+\frac{2}{a}\right) e^{-\frac{a}{2}(x-t)}-\frac{2}{a}\right) c D_{0 t}^{\alpha} u(t, 0) d t,
$$

is a solution of the equation (1), as for equation with right side at $a^{2}=4 c \neq 0$, where $z(x, y)$ is a solution of the equation (5), and the function:

$$
u(x, y)=-\frac{2}{a} \int_{0}^{x}\left(\left(x-t+\frac{2}{a}\right) e^{-\frac{a}{2}(x-t)}-\frac{2}{a}\right) c D_{0 t}^{\alpha} u(t, 0) d t,
$$

is a partial solution to the equation (1). Hence, (1) highlights the validity of the representation $(4)$, i.e. $u(x, y)=$ $z(x, y)+w(x)$ 
It follows from the latter representation that $u(x, 0)=z(x, 0)+w(x)$. Thus, from equation (7), it provides:

$$
w^{\prime \prime \prime}(x)+a w^{\prime \prime}(x)+c w^{\prime}(x)-c D_{0 x}^{\alpha} w(x)-c D_{0 x}^{\alpha} z(x, 0)=0,
$$

and the function $z(x, y)=u(x, y)-w(x)$, satisfies equation: (5) for $y>0$. In cases $a^{2}>4 c$ and $a^{2}<4 c$ respectively. Analogously, it was proved in the case for $y<0$. Theorem 1 is thus proved.

Remark 1. Taking into account, that the function $z(x)=\tilde{a} e^{\sqrt{\lambda} x}+\tilde{b} e^{-\sqrt{\lambda} x}+\tilde{c}$ satisfies the equation (5), for the investigation of the problem $D_{1}$, we can assume without loss of generality that

$$
w(0)=w^{\prime}(0)=w^{\prime \prime}(0)=0 .
$$

Denoting $w^{\prime}(x)=y(x)$ in the equation (6) we have:

$$
y^{\prime \prime}(x)+a y^{\prime}(x)+c y(x)-\frac{1}{\Gamma(1-\alpha)} \int_{0}^{x}(x-t)^{-\alpha} y(t) d t=c D_{0 x}^{\alpha} z(x, 0) .
$$

The characteristic equation, corresponding to the homogeneous equation, has the form

$$
k^{2}+a k+c=0
$$

Let us introduce the notation $\Delta=a^{2}-4 c$ :

1) If $\Delta=0$ then, the equation (10) has two real roots, moreover they are equal. Thus, at $a^{2}>4 c$ the equation (9) equivalent to the following form:

$$
\begin{aligned}
& y(x)-\frac{1}{\Gamma(1-\alpha)} \int_{0}^{x} y(t) d t \int_{t}^{x}(x-s)(s-t)^{-\alpha} e^{-\frac{a}{2}(x-s)} d s= \\
= & l_{1}(0) e^{-\frac{a}{2} x}+l_{2}(0) x e^{-\frac{a}{2} x}+\int_{0}^{x}(x-t) e^{-\frac{a}{2}(x-t)} c D_{0 x}^{\alpha} z(t, 0) d t,
\end{aligned}
$$

where $l_{1}(0)$ and $l_{2}(0)$ are unknown constants to be defined. In equation (9) at $s=t+(x-t) v$, with respect to $y^{\prime}(0)=y(0)=0$, we obtain the Volterra integral equation relative to $y(x)$ :

$$
y(x)-\int_{0}^{x} K_{1}(x, t) y(t) d t=\int_{0}^{x}(x-t) e^{-\frac{a}{2}(x-t)} c D_{0 x}^{\alpha} z(t, 0) d t,
$$

where:

$$
K_{1}(x, t)=\frac{(x-t)^{2-\alpha}}{\Gamma(1-\alpha)} \int_{0}^{1} v^{-\alpha}(1-v) e^{-\frac{a}{2}(x-t)(1-v)} d v .
$$

Solving the next integral equation (12) we obtain:

$$
y(x)=\int_{0}^{x}\left(K_{1}(x, t)+\int_{t}^{x} R_{1}(x, s) K_{1}(s, t) d s\right) z^{\prime}(t, 0) d t,
$$

where $R_{1}(x, s)$ is the resolvent of the kernel $K_{1}(x, t)$.

Hence, by virtue of the (8), (13) and the designation $w^{\prime}(x)=y(x)$, after some transformation, we have:

$$
w(x)=\int_{0}^{x} K(x, t) z^{\prime}(t, 0) d t
$$

where:

$$
K(x, t)=\int_{t}^{x}\left\{K_{1}(s, t)+\int_{t}^{s} R_{1}(s, z) K_{1}(z, t) d z\right\} d s .
$$

Similarly we can receive in the cases 2) $a^{2}>4 c$, and 3) $a^{2}<4 c$. 


\section{The main results}

Theorem 2. If $\psi_{1}^{\prime}(0)+2 \varphi_{1}(0)=\sqrt{2} \psi_{2}(0)$, and $\varphi_{1}(x) \in C^{1}[0,1) \cap C^{2}(0,1)$,

$$
\psi_{1}(x) \in C^{1}\left[0, \frac{1}{2}\right] \cap C^{3}\left(0, \frac{1}{2}\right), \quad \psi_{2}(x) \in C[0,1 / 2] \cap C^{2}(0,1 / 2),
$$

then there exists a unique solution to the problem $D_{1}$ in the domain $\Omega$.

Proof. Firstly, by virtue of the representation (4) and in view of (8), the equation (1) and boundary-value conditions (2), (3), are reduced to the form (5):

$$
\begin{gathered}
z_{y}(x, 0)=\varphi_{1}(x), \quad 0 \leq x<1, \\
z(x,-x)=\psi_{1}(x)-\int_{0}^{x} K(x, t) z^{\prime}(t, 0) d t, \quad 0 \leq x \leq \frac{1}{2}, \\
\frac{\partial z(x,-x)}{\partial n}=\psi_{2}(x)-\frac{1}{\sqrt{2}} \int_{0}^{x} K^{\prime}(x, t) z^{\prime}(t, 0) d t, \quad 0 \leq x \leq \frac{1}{2} .
\end{gathered}
$$

Since, problem $D_{1}$ was reduced to the equivalent non-local problem for a third order equation of mixed type (5), we may conclude that Problem $D_{1}$ has a unique solution, as a direct result of the unique solvability of the non-local problem.

Bearing in mind [15], after integration of the equation (5), with respect to $x$, we have the following form:

$$
z_{x x}-z_{y y}+a z_{x}+b z_{y}+c z=\widetilde{\omega}(y), \quad y<0,
$$

where $\widetilde{\omega}(y)$ is arbitrary continuous function.

First, we find the main functional relations on $I$ deduced from the domain $\Omega$. We introduce the following notation:

$$
\left.z(x, y)\right|_{y=-0}=\tau(x), \quad(x, 0) \in \bar{I},
$$

where $\tau(x)$ is unknown function.

The solution of the Cauchy problem for the equation (19) with the conditions (2), (20) can be represented in the form:

$$
\begin{gathered}
z(x, y)=\frac{1}{2}\left[\tau(x-y) \exp \left(-\frac{a-b}{2} y\right)+\tau(x+y) \exp \left(-\frac{a+b}{2} y\right)\right]- \\
\left.-\frac{1}{2} \exp \left(\frac{b}{2} y\right) \int_{x-y}^{x+y}\left(\frac{b}{2} J_{0}\left[\sqrt{\lambda\left((x-\xi)^{2}-y^{2}\right)}\right)\right]-\lambda y \hat{J}_{1}\left[\sqrt{\lambda\left((x-\xi)^{2}-y^{2}\right)}\right]\right) \times \\
+\frac{1}{2} \exp \left(\frac{b}{2} y\right) \int_{x-y}^{x+y} J_{0}\left[\sqrt{\lambda\left((x-\xi)^{2}-y^{2}\right)}\right] \exp \left(-\frac{a}{2}(x-\xi)\right) \varphi_{1}(\xi) d \xi+ \\
+\frac{1}{2} \int_{0}^{y} \widetilde{\omega}(\eta) d \eta \int_{x-y+\eta}^{x+y-\eta} J_{0}\left[\sqrt{\lambda\left((x-\xi)^{2}-(y-\eta)^{2}\right)}\right] d \xi \\
\lambda=\frac{1}{4}\left(4 c^{2}-a^{2}-b^{2}\right),
\end{gathered}
$$

where $J_{0}[z], J_{1}[z]$ is Bessel function, $\hat{J}_{1}[z]=J_{1}[z] / z$.

Then using condition (18) from (21), taking account of $\tau^{\prime}(0)+\varphi_{1}(0)=\sqrt{2} \psi_{2}(0)$ and considering of the property of the Bessel functions and by replacing the argument $x$ for $-y$ in the relation, we get the following functional relation, transferred from the domain $\Omega$ to $I$ :

$$
\begin{gathered}
\widetilde{\omega}(y)+\lambda_{2} \int_{0}^{y} L(\eta, y) \widetilde{\omega}(\eta) d \eta=\Phi^{\prime}(y), \\
L(\eta, y)=\int_{\eta-2 y}^{-\eta}\left[\hat{J}_{1}[z(-y, y ; \xi, \eta)]-\frac{1}{2} J_{2}[z(-y, y ; \xi, \eta)]\right] d \xi,
\end{gathered}
$$




$$
\begin{gathered}
\Phi(y)=\sqrt{2} \psi_{2}(-y)-w^{\prime}(-y)+\frac{a-b}{4} \tau(-2 y) \exp \left(-\frac{a-b}{2} y\right)- \\
-\frac{1}{2} \exp \left(\frac{a+b}{2} y\right) \int_{-2 y}^{0}\left[p_{0}(\xi, y) \tau(\xi)+p_{1}(\xi, y) \varphi_{1}(\xi)\right] d \xi- \\
-\left[\varphi_{1}(0)+\frac{2 \lambda y+a-b}{4} \tau(0)+\tau^{\prime}(0)\right] \exp \left(\frac{a+b}{2} y\right), \\
z(x, y ; \xi, \eta)=\sqrt{\lambda\left((x-\xi)^{2}-(y-\eta)^{2}\right)}, z(x, y ; \xi, 0)=z(x, y ; \xi) .
\end{gathered}
$$

Both $p_{0}(\xi, y), p_{1}(\xi, y)$ are known continuous functions. Hence, if we reckon the right-hand side known, with regards the properties of the special functions, and the theory of the integral equations of the Volterra type, the solution of the equation (22) can be written in the form:

$$
\widetilde{\omega}(y)=\Phi^{\prime}(y)+\int_{0}^{y} R_{2}(y, t) \Phi^{\prime}(t) d t
$$

here $R_{2}(y, t)$ is the resolvent of the kernel $L(\eta, y)$.

Further, from (21) bearing mind (17), $\tau(0)=\psi_{1}(0)$, we deduce the next functional relation, transferred from the $\Omega$ to $I$ :

$$
\begin{gathered}
\tau(x)+\int_{0}^{x} \tilde{p}_{0}\left(\frac{x}{2}, \xi\right) \exp \left(-\frac{a}{2}(x-\xi)\right) \tau(\xi) d \xi- \\
-\int_{0}^{x} J_{0}\left[z\left(\frac{x}{2},-\frac{x}{2} ; \xi\right)\right] \exp \left(-\frac{a}{2}(x-\xi)\right) \varphi_{1}(\xi) d \xi= \\
=-\psi_{1}(0) \exp \left(-\frac{a}{2} x\right)+2 \exp \left(-\frac{a-b}{4} x\right) \times \\
\times\left\{\psi_{1}\left(\frac{x}{2}\right)-\omega\left(\frac{x}{2}\right)-\frac{1}{2} \int_{0}^{-\frac{x}{2}} \tilde{\omega}(\eta) d \eta \int_{x+\eta}^{-\eta} J_{0}[z(x,-x ; \xi, \eta)] d \xi\right\} .
\end{gathered}
$$

The equality (25) with regards (24), is equivalent to the system of integral equation with shift. We can easily obtain the Volterra integral equation with shift regarding the unknown function $\tau^{\prime}(y)$, transferred from the domain $\Omega$ to $I$. Hence, taking account of (15) and based on the general theory of integral equations, one can easily be sure that equation has a unique solution.

Thus, solution of the problem $D_{1}$ in the domain $\Omega$ in view of (14), (24) and (25) is determined uniquely according to the formula (4). Therefore, we can conclude that, there exists a regular solution of problem $D_{1}$. Thus, the constructed solution of the problem $D_{1}$ is unique. Indeed, let $\varphi_{1}(x)=\psi_{1}(x)=\psi_{2}(x)=0$, then from (14) (respectively (24) and (25)), we have $w(x)=0$ and $\tau(x)=0$. It follows from this and (21) that $\mathrm{z}(x, y) \equiv 0$ in $\bar{\Omega}$. Consequently, the homogeneous boundary value problem has no nontrivial solutions, i.e. from formulas (4), we have $\mathrm{u}(x, y) \equiv 0$ in the domain $\Omega$, Q.E.D.

Thus, we have proved that Problem $D_{1}$ is uniquely solvable.

For example, let $a=c=0$ in the equation (1), in that case, the kernel of the equation (12) has the form $K_{1}(x, t)=\frac{(x-t)^{2-\alpha}}{\Gamma(3-\alpha)}$ and we can find the resolvent of the kernel as:

$$
R_{1}(x, t)=\sum_{i=1}^{n} \frac{(x-t)^{(3-\alpha) i-1}}{\Gamma((3-\alpha) i)} .
$$

If we can choose the given functions taking into account the conditions of Theorem 2, applying $z(x, y)=x-y$, then we obtain the solution of the problem (6) and (8) in the form:

$$
w(x)=\frac{x^{4-\alpha}}{\Gamma(5-\alpha)}+\sum_{i=1}^{\infty} \frac{x^{4-\alpha+(3-\alpha) i}}{\Gamma(5-\alpha+(3-\alpha) i)}, \quad 0<\alpha<1 .
$$

Consequently, we can find the exact solution to the problem (1), (2) and (3), which is represented in the Fig. 1.

Similarly, we can prove solvability of this analogous problem Darboux $D_{2}$. 


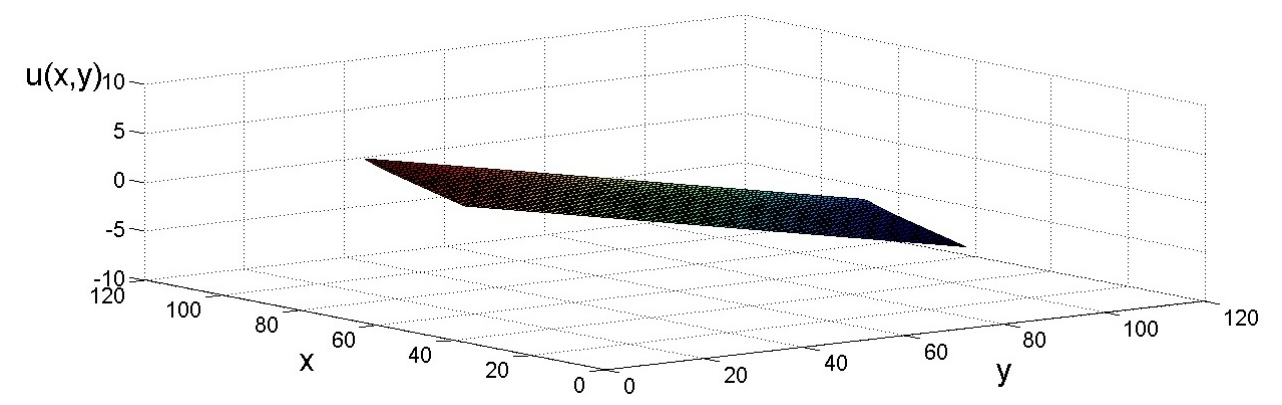

FIG. 1. Exact solution to the problem (1), (2) and (3)

Problem $\mathbf{D}_{2}$. Find a regular solution $u(x, y)$ equation (1) in $\Omega$, which has continuous derivatives $u_{x}\left(u_{y}\right)$, up to $A B \cup B C(B C)$, and satisfies the conditions (2):

$$
u(x, x-1)=\psi_{3}(x),\left.\quad \frac{\partial u(x, y)}{\partial n}\right|_{y=x-1}=\psi_{4}(x), \quad \frac{1}{2} \leq x \leq 1,
$$

where $\psi_{3}(x)$ and $\psi_{4}(x)$ are given real-valued functions.

Theorem 3. If $\psi_{3}^{\prime}(1)=-\sqrt{2} \psi_{4}(1)+2 \varphi_{2}^{\prime}(0)$, and the conditions (15) and:

$$
\psi_{3}(x) \in C^{1}[1 / 2,1] \cap C^{3}(1 / 2,1), \psi_{4}(x) \in C[1 / 2,1] \cap C^{2}(1 / 2,1)
$$

are satisfied, then there exists a unique solution to the problem $D_{2}$ in the domain $\Omega$.

Theorem 3 is proved in the same way as the Theorem 2 . That is, by virtue of the Theorem 1, equation (1) and the conditions (2), (16), in view of (8), are reduced to the form (5), (16) and:

$$
\begin{gathered}
z(x, x-1)=\psi_{3}(x)-w(x), 0 \leq x \leq \frac{1}{2} \\
\left.\frac{\partial z(x, y)}{\partial n}\right|_{y=x-1}=\psi_{4}(x)-\frac{1}{\sqrt{2}} w^{\prime}(x), \quad 0 \leq x \leq \frac{1}{2},
\end{gathered}
$$

where $w(x)$ expressed in terms of (14).

Since, problem $D_{2}$ was reduced to the equivalent non-local problem. The equation (5) can be represented in the form (19). Thus, using the solution of the Cauchy problem (21), taking into account (18):

$$
\tau(1)=\psi_{3}(1), \tau(x)=\psi_{3}(1)-\int_{x}^{1} \tau^{\prime}(t) d t, \quad \psi_{3}^{\prime}(1)=-\sqrt{2} \psi_{4}(1)+2 \varphi_{1}^{\prime}(0),
$$

we obtain the first functional relation, transferred from the $\Omega$ to $I$. On the other side, the relation (25) between the functions $\tau(x)$ and $\varphi_{1}(x)$ transferred from the $\Omega$ to $I$ is correctly. Further, conducting an analogous reasoning to that of $\tau(x)$, from the main functional relations with regards of conditions of the problem, we can find the $\tau(x)$ and $w(x)$. The subsequent investigations are performed by analogy with problem $D_{1}$.

\section{Conclusion}

One of the directions of the modern theory of partial differential equations, which has been rapidly developing in recent years, is the theory of nonlocal problems. In this paper, we investigated solvability of problems for linear loaded integro-differential equations. Taking into account Theorem 1, our problem reduced to nonlocal problems for partial differential equations with conditions (16)-(18) and (16), (27)-(28). Attention to such problems is not due to only theoretical interest, but also practical necessity. Mathematical modeling a number of physical and biological processes is of interest to modern natural science as nanosciences, nano-engineering.

We should note that the problem under consideration has investigated the equation with a wave operator which appears in problems of nanophysics and is present in some micro and nanoflow models of spinodal decomposition models, and also described flow in thin viscous layers subjected to ultrasonic acoustic field and etl [17-21].

Results [22] show that for tension bars in small length scales, nonlocal effects would have significant influences on the study of nano-structures and nonlocal theory could potentially play a useful role in analysis related to nanotechnology applications [22]. In prior studies [23], nonlocal differential problem, supplemented with non-standard boundary conditions, is highlighted and shown to lead to mathematically well-posed problems of nano-engineering. 
The theoretical predictions, exhibiting stiffening nonlocal behaviors, are therefore appropriate to significantly model a wide range of small-scale devices of nanotechnological interest [23].

In the work [24] researchers considered a general form of fractional integro-differential equations arising in nanotransistors, which were described with loaded differential equation.

As noted in other studies [25,26], many important materials used in modern technologies (such as nanotechnology) are viscoelastic and anisotropic. In mathematical modeling of processes taking place in viscoelastic materials, there is a so-called system with memory, whose behavior is not completely determined by the state at the moment, but depends on the systems of entire history, and therefore, describes an integro-differential equation which contains the corresponding integral with respect to the time variable. The equation (1), taking into account the integro-differential operator is basic in the linear theory of viscoelastic anisotropic media [26].

\section{References}

[1] Nakhushev A.M. Equations of mathematical biology. Vishaya shkola, Moscow, 1995, 301 p.

[2] Serbina L. I. Nonlocal mathematical models of movement in watertransit systems. Moscow, Nauka, 2007.

[3] Berdyshev A. S. The basis property of a system of root functions of a nonlocal problem for a third-order equation with a parabolic-hyperbolic operator. Diff Equat, 2000, 36, P. 417-422.

[4] Sabitov K.B. Nonlocal problem for a parabolic-hyperbolic equation in a rectangular domain.Math Notes, 2011,89 , P. $562-567$.

[5] Zikirov O. S. A nonlocal boundary value problem for third order linear partial differential equation of composite type. Math. Model. And Anal., 2009, 14(3), P. 407-421.

[6] Belakroum Kh., Ashyralyev A., Guezane-Lakoud A. Stability of boundary-value problems for third-order partial differential equations. Electronic Journal of Differential Equations, 2017, 2017(53), P. 1-11.

[7] Khashimov A. R., Smetanov D. Nonlocal Problem for a Third-Order Equation with Multiple Characteristics with General Boundary Conditions. Axioms, 2021, 10(2), P. 110.

[8] Fedorov Valery E. and Efimova Elena S. Nonlocal boundary value problems for a third order equation of a composite type. AIP Conference Proceedings, 2021, 2328, 020009.

[9] Baltaeva U. I. The loaded parabolic-hyperbolic equation and its relation to non-local problems. Nanosystems physics, chemistry, mathematics, 2017, 8(4), P. 413-419.

[10] JinRong Wang, Yong Zhou, Wei Wei, Honglei Xu. Nonlocal problems for fractional integrodifferential equations via fractional operators and optimal controls. Computers and Mathematics with Applications, 2011, 62(3), P. 1427-1441.

[11] Chang Y.K., Kavitha V., Mallika Arjunan M. Existence and uniqueness of mild solutions to a semilinear integrodifferential equation of fractional order. Nonlinear Anal., 2009, 71, P. 5551-5559.

[12] Jin Rong Wang, Yong Zhou A. class of fractional evolution equations and optimal controls. Nonlinear Anal., 2011 , 12, P. $262-272$.

[13] Islomov B, Baltaeva U. I. Boundary value problems for a third-order loaded parabolic-hyperbolic equation with variable coefficients. Electronic Journal of Differential Equations, 2015, 221, P. 1-10.

[14] Atangana A. Derivative with a New Parameter, Editor(s): Abdon Atangana. Chapter 1 - History of derivatives from Newton to Caputo Academic Press, 2016, P. 1-24.

[15] Baltaeva U. Solvability of the analogs of the problem Tricomi for the mixed type loaded equations with parabolic-hyperbolic operators. Boundary Value Probl., 2014, 211.

[16] Abdullaev O. K., Islomov B. I. Gellerstedt Type Problem for the Loaded Parabolic-Hyperbolic Type Equation with Caputo and Erdelyi-Kober Operators of Fractional Order. Russ Math., 2020, 64, P. 29-42.

[17] Kirillova S. A., Almjashev V. I., Gusarov V. V. Spinodal decomposition in the $\mathrm{SiO}_{2} \mathrm{TiO}_{2}$ system and hierarchically organized nanostructures formation. Nanosystems: Physics, Chemistry, Mathematics, 2012, 3(2), P. 100-115.

[18] Antonov N. M., Popov I. Yu., Gusarov V. V. Model of spinodal decomposition of phases under hyperbolic diffusion. Physics of the Solid State, 1999, 41(5), P. 824-826.

[19] Lobanov I. S., Nikiforov D. S., Popov I. Y. et al. Model of time-dependent geometric graph for dynamical Casimir effect. Indian J. Phys., 2020.

[20] Melikhov I. F., Popov I. Y. Model of cell membrane in ultrasonic field. Chinese Journal of Physics, 2020, 65, P. 334-340.

[21] Agarwal P., Baltaeva U., Alikulov Y. Solvability of the boundary-value problem for a linear loaded integro-differential equation in an infinite three-dimensional domain. Chaos, Solitons and Fractals, 2020, 140, P. 110108.

[22] Tepe Aysegul, Artan, Reha, Application of Nonlocal Bars in Tension to Nanotechnology. Journal of Computational and Theoretical Nanoscience, 2009, 6(3), P. 537-540.

[23] Raffaele Barretta, Francesco Marotti de Sciarra, Marzia Sara Vaccaro On nonlocal mechanics of curved elastic beams. arXiv:2009.09188v1 [physics.app-ph] 19 Sep. 2020.

[24] Avaji M., Dehcheshmeh S. S., Hafshejani J. S., Ghahfarokhi D.F. On the Solution of Linear and Nonlinear Fractional Integro-Differential Equations in Nano-Transistors. Journal of Computational and Theoretical Nanoscience, 2013, 10, P. 510-513.

[25] Baleanu D., Tenreiro Machado J. A., Guvenc Z. B. New Trends in Nanotechnology and Fractional Calculus Applications. Springer-Verlag, London, 2010.

[26] Durdiev D. K., Durdiev U. D. The problem of kernel determination from viscoelasticity system integro-differential equations for homogeneous anisotropic media. Nanosystems: Physics, Chemistry, Mathematics, 2016, 7(3), P. 405-409. 